\title{
LA CONFIGURACIÓN ESPACIAL DEL DESARROLLO REGIONAL FROTERIZO
}

\author{
Por \\ Victor M. Castillo*
}

\begin{abstract}
RESUMEN
En este trabajo el autor plantea la idea de que, antes del periodo de consolidación (1960-1980), en la frontera norte de México ya se había configurado el modelo de desarrollo económico como una economía primaria-terciarizada; mientras que durante las décadas de los sesenta y setenta se consolidó el desarrollo del sector secundario. Asimismo afirma que, contrariamente a algunas opiniones, la migración y el PIF no establecieron las condiciones necesarias para el desarrollo, aunque si fueron agentes que aceleraron dicho proceso.
\end{abstract}

\begin{abstract}
In this work the author proposes the idea that before the consolidation period (1960-1980), the model of economic development of a primary-terciarized economy had already been established in Mexico's Northem border, while in the sixties and seventies the secondary sector development was consolidated. Also, the author afirms that contrary to some opinios, inmigration and PIP did not established the conditions needed for development, eventhough they actually were accelerating agents for it.
\end{abstract}

\section{INTRODUCCIÓN: HACIA UNA CONCEPCIÓN REGIONAL DE LA FRONTERA NORTE}

El objetivo del presente trabajo es entender la conformación del desamollo regional de la frontera norte de México durante el periodo de 1960 - 1980. Se acepta que antes de 1960, la formación regional característica del periodo se había ya alcanzado. Quiere decir que la base económica rogional de la zona fronteriza se desarrolló a lo largo de un extenso periodo histórico que va, en esencia, de principios del siglo XX hasta los años mesenta. De esta manera, el periodo moderno de 1960 a 1980 es de consolidación del extenso desenvolvimiento regional que ya había tenido lugar en la región en su periodo previo, a pesar de que tradicionalmente se considera que tanto la migración mexicana hacia los Estados Unidos como

- Profesor asociado, Facultad de Economía, UABC. 
el Programa de Industrialización Fronteriza (PIF), han sido los promotores básicos de la formación regional de la frontera norte durante los últimos veinte afios. Se enfatiza entonces, que estos dos procesos de índole internacional sólo han contribuido en este periodo a reafirmar la consolidación del proceso regional que la frontera del norte ya había establecido, y que no fueron, como se propone, los promotores de su desarrollo.

En este marco se busca establecer el porqué y los límites en los cuales se puede identificar una región fronteriza común y no común en el norte de México. Son variados los estudios que existen en este ámbito. Sin embargo, se encuentran límites en cuanto a una concepción regional única de la frontera norte de México. Estos estudios han abundadoen caracterizar a la frontera del norte de México en términos de zonas fronterizas; tal es el caso de integrar solamente los municipios fronterizos que colindan con los municipios fronterizos del suroeste de los Estados Unidos. Otros han integrado los estados fronterizos del norte de México y los han llamado también zona fronteriza. Existen aquéllos que plantean que todos los estados del norte de México no representan una región homogénea, y que el simple hecho de que se encuentren geográficamente colindantes con la frontera sur de los Estados Unidos no les hace conformar una región homogénea prototipo. Por el contrario, se entiende que la larga frontera del norte del país está integrada por varias subregiones que tienen en común la ubicación geográfica. Esta es en sí la discusión del presente trabajo, pues como se ha mencionado, se trata de identificar los términos en los que se entiende la existencia de una región fronteriza en el norte de México y cuáles serían los indicadores que le confieren dicha estructura.

\section{EL ESTUDIO DEL DESARROLLO REGIONAL EN MÉXICO}

La historia espacial de México es una historia de desigualdades sectoriales y regionales. Históricamente el país se ha conformado de manera desigual y regionalmente dividido. La conformación regional se caracteriza por ser la de un desarrollo geográfico desigual. Esta caracterización explica de una manera mejor y funcional las intrínsecas diferencias regionales del pars. México es un país de variados contrastes, cuyo tamaño, en comparación al de Estados Unidos, es casi una cuarta parte; es el tercer país más grande de la América Latina; y aunque la estructura regional de México es una cuestión debatible, múltiples alternativas acerca de la división regional en México se han propuesto desde los pioneros estudios sobre regionalismo en México. Entre las primeras divisiones regionales propuestas se encuentra la de Stern (1967); estudios posteriores sobre 
regionalización (Stern, 1973; Unikel y Victoria, 1970) proponían un marco espacial formado en términos de agregaciones por estados y construido bajo el sistema urbano nacional existente. La estructura regional era usualmente determinada por la ubicación geográfica, el medio ambiente natural, las características de la población, así como por las condiciones económicas. Los indicadores regionales propuestos eran: producción industrial, empleo en la industria, capital invertido en la producción industrial, consumo per cápita, distribución de los salarios mínimos, mortalidad infantil y analfabetismo entre otros indicadores sociales.

Otro tipo de estudio regional sobre México conservaba interés en la identificación y proposición de una división regional para el país, basada en una regionalización por estados y por nuevos indicadores de regionalización (Bataillon, 1969; CEPAL, 1972; Unikel, 1976; Barkin y King, 1970; Bassols Batalla, 1971, 1972, 1979 y 1982; Lavell, 1972 y COPLAMAR, 1982); las nuevas divisiones regionales propuestas para el país resultaron con opiniones divergentes. Algunas dividían al país en ocho regiones diferentes, con diferencia sólo en las inclusiones de los estados (Unikel, 1976; Bassols Batalla, 1971), otras propuestas lo hacian en doce regiones (COPLAMAR, 1982). Una buena introducción a las diferencias con respecto a las divisiones regionales propuestas, es la proporcionada por COPLAMAR (1982). ${ }^{1}$

\section{DESARROLLO REGIONAL Y FRONTERA NORTE}

La división regional propuesta por COPLAMAR es la más cercana a considerar a la mayoría de los estados de la frontera norte de México como una misma región. Es común encontrar estudios fronterizos que consideran tanto a los estados como a los municipios fronterizos del norte del país como una región (Urquidi y Méndez Villareal, 1978; Tamayo, 1983), aunque esta perspectiva no es compartida por la mayoría de los estudios

1 La división regional elaborada por CoPLAMNR para México sigue la siguiente clasificación:

I Pacifico sur: Chiapas, Guerrero, Oaxaca.

II Centro este: Hidslgo, Puebla, Thaxcala.

mi Centro norte: San Luis Potosi, Zacatecas.

rv Centro occidente: Guanajuato, Michoacán, Querétaro.

v Centro golfo: Tabasco, Veracruz.

vi Sureste: Campeche, Quintana Roo, Yucatan.

va Pacifico centro: Durango, Nayarit, Sinaloa.

vim Centro: México, Morelos.

xx Occidente: Aguascalientes, Colims, Jalisco.

$x$ Norte: Coahuila, Chihuahua, Nuevo León, Tamaulipas.

xı Pacifico norte: Baja Califormis, Baja Califomia Sur,

XII Distrito Federal. 
regionales relevantes hechos en México. Por el contrario, hay argumentos en contra de esta concepción. Un acercamiento alternativo, que es más congruente con la división regional propuesta, ha sido el de considerar la frontera de México como un grupo de regiones o subregiones (Victoria, 1982; Security Pacific National Bank, 1981).

El argumento principal en contra de la idea de que los estados del norte de México forman una región, se basa en la existencia de importantes diferencias entre los estados del norte, lo que dificulta el pensar que los estados fronterizos del norte componen una región integral y homogénea. No obstante estos hechos, existen estudios e información empírica disponibles que hacen posible argumentar que los estados del norte de México y los municipios fronterizos del norte comparten similitudes económicas y sociales únicas (o particulares). ${ }^{2}$ La prolongada existencia de las zonas y perímetros libres y la continua y extensiva aplicación de políticas especiales para la frontera, implementadas por el gobierno mexicano desde los años sesenta a través de diferentes programas fronterizos, han diferenciado el desarrollo fronterizo del desarrollo del resto del país. El desarrollo de los estados del norte y la franja fronteriza han seguido un patrón de crecimiento más homogéneo en comparación con otras regiones de México. Otras experiencias regionales comparables, que han sido objeto de una participación mayor de la intervención del Estado, lo fueron el desarrollo de las cuencas hidrológicas en México y los considerables efectos negativos del impacto regional de la explotación petrolera en la región de las costas del Golfo de México.

Los cambios en la estructura del empleo es otro indicador de esta homogeneidad en la región del norte. Un patrón similar es el que se observa tanto en los estados del norte del país como en dos municipios fronterizos a los que se toman como ejemplo durante el periodo de 1960 a 1980. Ambos municipios mostraron un decremento en las actividades agrícolas comparadas con el resto del país. La proporción de actividad manufacturera está considerablemente por encima del nivel nacional. En general, los estados fronterizos del norte de México muestran una economía basada en los sectores secundario y terciario, mientras que a nivel nacional el sector primario predomina sobre los otros dos. Estos indicadores son evidencia de que existe una región fronteriza del norte, aunque también sugieren que el norte de México es, en efecto, una región heterogénea cuando se escudrifia en sus particularidades.

2 Comúnmente es reconocido que los estados del norte de México mantienen los más altos estándares de bienestar en el país. Los estudios hechos por COPLAMAR verifican que los niveles de bienestar en los estados del norte de México eran entre los más altos del país y sólo están por debajo del Distrito Federal (COPLAMAR, 1979, 1982). 
Entre los factores específicos más importantes que promueven la idea de la frontera mexicana como un área especial de desarrollo regional se encuentran: primero, la tendencia del acelerado crecimiento demográfico que se inició en los affos treinta, cuando México comenzó a consolidarse come unidad territorial (Alba, 1982). Los estados fronterizos para el periodo 19601970 , según se muestra en el cuadro 1, observaron tasas de crecimiento altas, muchas de ellas igual o por encima de la tasa de crecimiento nacional para el mismo periodo. ${ }^{3}$ En cuanto al segundo factor, concerniente al desarrollo de la región fronteriza mexicana, algunos autores sostienen que éste se ha debido a tres situaciones 1) el rápido crecimiento poblacional en la frontera; 2) la fuerza de trabajo que emigra hacia los Estados Unidos; 3) la política económica

CUADRO 1. Población total y tasas anuales de crecimiento (TAC) para México, estados fronterizos del norte de México, Tijuana y Ciudad Juárez 1960-1980 (en miles).

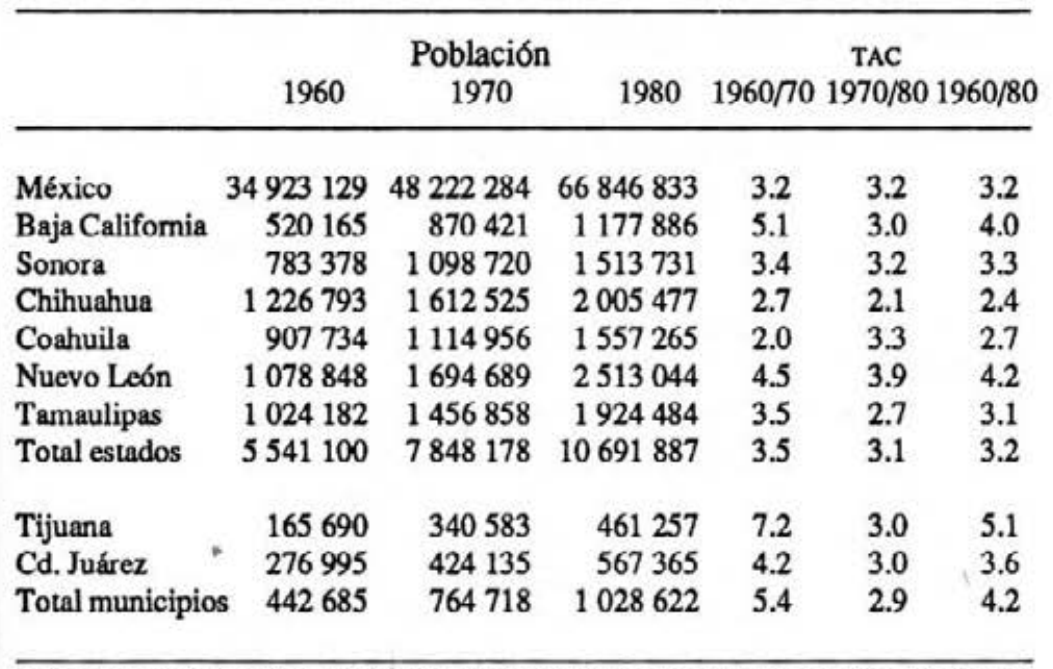

FUENTE: VIIICenso General de Población, 1960. Dirección General de Estadística, SIC DX Censo general de población 1970. Dirección general de estadística, SIC, X Censo general de población, 1980. SPP-INEGI. Volúmenes de Baja California y Chihuahua; 1960, 1970, 1980.

NOTA: Tasas calculadas por el autor.

3 Se ha documentado ampliamente que después de los setenta, la región fronteriza del norte de México mantenía un acelerado ritmo de crecimiento (Victoria Mascorro, 1982); esto es confirmado por los cuadros incluidos en el texto (ver cuadro 1) para el periodo de $1970 \mathrm{a}$ 1980. Las tasas anuales de crecimiento para ese periodo muestran una magnitud menor o igual a las reportadas nacionalmente. 
aplicada, fundamentalmente por el impulso de la industria de ensamble de la frontera (Alba, 1982). El tercer factor se refiere a la proximidad del área fronteriza con los Estados Unidos.

México, país en vías de desarrollo, alguna vez clasificado como parte de los nuevos países industrializados (NICSs), comparte una frontera de 1,936 millas desde el océano Pacífico hasta el golfo de México. Seis estados mexicanos del norte, con el 16 por ciento del total de la población del país en 1980, componen la frontera México-Estados Unidos junto con cuatro estados del sur de Estados Unidos, que a su vez concentraban el 18.5 por ciento de la población total de ese país en el mismo año.

Esta proximidad física ha creado interrelaciones sociales, políticas, económicas y territoriales, a pesar de las diferencias existentes en los niveles de desarrollo y organización social en ambos lados de la frontera (Alba, 1982; Bustamante, 1979; Graizbord y Hiernaux, 1981; Tamayo y Fernández, 1983; Stoddard, 1975-76). Pero precisamente por esta convergencia espacial es que dichas relaciones se han expresado históricamente con elementos de fricción y conflictos potenciales. Variadas perspectivas

CUADRO 2. Población total y tasas anuales de crecimiento (TAC) para los Estados Unidos, los estados del suroeste y los condados de San Diego y El Paso (miles).

\begin{tabular}{|c|c|c|c|c|c|c|}
\hline \multirow[b]{2}{*}{ Estados Unidos } & \multicolumn{3}{|c|}{ Población } & \multicolumn{3}{|c|}{$\begin{array}{c}\text { TAC } \\
1960 / 701970 / 801960 / 80\end{array}$} \\
\hline & 179977 & 20505 & 2227704 & 1.3 & 3.0 & 1.1 \\
\hline California & 15850 & 20023 & 23771 & 2.3 & 1.7 & 2.0 \\
\hline Arizona & 1318 & 1795 & 2731 & 3.0 & 4.2 & 3.6 \\
\hline Nuevo México & 958 & 1023 & 1305 & 0.6 & 2.4 & 1.5 \\
\hline Texas & 9617 & 11244 & 14321 & 1.5 & 2.4 & 1.9 \\
\hline Total estados & 27743 & 34085 & 42128 & 2.0 & 2.1 & 2.0 \\
\hline San Diego & 1033 & 1358 & 1862 & 2.7 & 3.1 & 2.9 \\
\hline El Paso & 314 & 359 & 480 & 1.3 & 2.9 & 2.1 \\
\hline Total condados & 1347 & 1717 & 2342 & 2.4 & 3.1 & 2.7 \\
\hline
\end{tabular}

FUENTE: U.S. Bureau of the Census, U.S. Census of Population, 1960; U.S. Bureau of the Census, US. Census of Population, 1970; U.S. Bureau of the Census, US. Census of Population, 1980; Vol. Characteristics of the Population, Detailed Population Characteristics for the three periods; Volúmenes específicos para California y Texas; 1960, 1970 and 1980. NOTA: Tasas calculadas por el autor. 
desde distintos campos de estudio han expresado esta convergencia; las más sobresalientes han sido en el campo del desarrollo económico (Tamayo, 1980, 1983); relaciones comerciales (James, 1983; Tamayo, 1981); relaciones industriales (Corona, 1983); ecología y recursos naturales (Ross, 1983); política y relaciones exteriores (México y Estados Unidos Grupo Interparlamentario, 1962-1982) y desarrollourbano y regional (Bustamante Lemus, 1981; Graizbord y Hiernaux, 1981). Además, la discusión se ha planteado en las relaciones entre la región fronteriza del norte de México y la región fronteriza del suroeste norteamericano; ésta a su vez se ha interpretado como relación transfronteriza (Hansen, 1983; Friedmann y Morales, 1984) e industrialización vía maquiladora (Alba, 1982; Fernández, 1973; Seligson y Williams, 1981).

En lo que respecta al campo de las relaciones que se establecen con los Estados Unidos y la frontera sur norteamericana, ha sido interpretada bajo la perspectiva de una relación de dependencia (Xirau y Díaz, 1976; Bustamante Lemus, 1981; Torres, 1976), articulación dependiente (Tamayo, 1983) e interdependencia (Flores Caballero, 1982; Graizbord, 1983; Victoria, 1982).

\section{FRONTERA NORTE: CAMBIO DEMOGRÁFICO}

El crecimiento poblacional de la frontera mexicana es impresionante. En cuarenta affos, dos de las más importantes ciudades de la frontera, Tijuana y Ciudad Juárez, han experimentado explosivas tasas de crecimiento (ver cuadro 3). Durante el periodo 1960 a 1980, la población de la región fronteriza ya se había estabilizado. Durante este periodo las tasas anuales de crecimiento poblacional estuvieron por debajo de las que se habían observado entre los años veinte a los sesenta. Los cuadros 1 y 2 muestran el cambio poblacional en México, los Estados Unidos, los estados fronterizos México-Estados Unidos y los pares de ciudades gemelas Tijuana-San Diego, Ciudad Juárez y El Paso. La población total estimada para México en 1980 era cercana a los 67 millones de habitantes; para los Estados Unidos era de 227. Los estados fronterizos del norte de México alcanzaron los 10.7 millones, mientras que los estados fronterizos del sur de los Estados Unidos llegaron a 42.1 millones de habitantes. A nivel de ciudades fronterizas, tanto Tijuana como Ciudad Juárez alcanzaron solamente el millón de habitantes mientras que San Diego y El Paso llegaron a 2.3 millones.

En el lado mexicano, durante 1960-1980 la tasa anual de crecimiento fue de $3.2 \%$, que es considerada alta. Los estados fronterizos del norte, a partir de 1960 , tuvieron una tasa de crecimiento de $3.5 \%$, para 1980 esta 
tasa se redujo al $3.2 \%$, lo que muestra que la tendencia de los estados fronterizos mexicanos ha sido hacia un crecimiento estable. En las ciudades fronterizas esta tendencia es aún más clara, dado que tradicionalmente mantenían su crecimiento por arriba de las tasas nacionales (ver cuadro 3 ). Por lo que respecta al lado de los Estados Unidos, el crecimiento ha sido considerablemente menor que en el lado mexicano. La tasa de crecimiento nacional de 1960 a 1980 fue solamente de $1.1 \%$ y la tasa de crecimiento en el mismo periodo para los estados del suroeste de los Estados Unidos fue del doble, es decir, un $2.0 \%$.

CUADRO 3. Población total 1930-1980 y tasas anuales de crecimiento (TAC) 1940-1980 para Ciudad Juárez y Tijuana (miles).

\begin{tabular}{|c|c|c|c|c|}
\hline \multirow[b]{2}{*}{ Affo } & \multicolumn{2}{|c|}{ Ciudad Juárez } & \multicolumn{2}{|c|}{ Tijuana } \\
\hline & Población & TAC & Población & TAC \\
\hline 1930 & 43138 & & 11271 & \\
\hline 1940 & 55024 & 2.4 & 21977 & 6.7 \\
\hline 1950 & 131308 & 8.7 & 65364 & 10.1 \\
\hline 1960 & 276995 & 7.4 & 165690 & 9.3 \\
\hline 1970 & 424135 & 4.2 & 340583 & 7.2 \\
\hline 1980 & 567365 & 3.0 & 461257 & 3.0 \\
\hline & $\begin{array}{l}x, \text { Censos } \\
\text { n General d } \\
\text { y Chihuah } \\
\text { alculadas po }\end{array}$ & $\begin{array}{l}\text { poblc } \\
\text { istadi }\end{array}$ & $\begin{array}{l}\text { 1940, 1950, } \\
\text { SPP-INEGI, V }\end{array}$ & $\begin{array}{l}60,1 \\
\text { imene }\end{array}$ \\
\hline
\end{tabular}

A nivel de condados, en San Diego y El Paso la tasa media fue de 2.7\% que es considerablemente superior; la tasa más alta fue mantenida por San Diego (2.9\%) y solamente $2.1 \%$ para El Paso, lo que indica un rápido crecimiento poblacional en la región del suroeste de los Estados Unidos.

Las principales conclusiones que se derivan de los cuadros 1 y 2 son: que las tasas de crecimiento de México indican que el área fronteriza del país está creciendo más rápidamente, aunque en ambos lados de la frontera ha comenzado un lento descenso en las tasas de crecimiento poblacional desde 1960; del cuadro 3 se puede concluir que aunque Ciudad Juárez tiene más población, Tijuana es todavía un fuerte polo de crecimiento poblacional. 


\section{FRONTERA NORTE: CRECIMIENTO ECONÓMICO}

Los cuadros 4 y 5 muestran la estructura del empleo en México y Estados Unidos de 1960 a 1980. Probablemente la tendencia más significativa de la información sobre México es la declinación de la agricultura desde 1960 hasta 1980 . Otros sectores económicos durante el periodo estudiado no muestran cambios o tendencias significativas. Lo que es común para la mayoría de los sectores es un crecimiento negativo durante los ochenta, lo que probablemente puede explicarse por la crisis económica más fuerte que ha sufrido México y que se ha mantenido durante la última década. La categoría "otros" llama la atención por su crecimiento. La explicación posible de esta situación tal vez sea la gran cantidad de trabajadores que se han concentrado en el sector informal de la economía por la severa crisis que atraviesa el país y por la falta de crecimiento significativo en el empleo. Consecuentemente, el subempleo se ha incrementado y la economía encubierta (o subterránea) se ha desarrollado; por ello, muchas relaciones de trabajo espontáneas se han convertido en actividades de trabajo generalmente aceptadas.

Respecto al empleo y la actividad económica para la frontera norte de México, el periodo de 1960 a 1980 es el punto inicial del desarrollo

CUADRO 4. México, estructura del empleo, 1960, 1970, 1980 (miles).

\begin{tabular}{lrrrrrr}
\hline Sector económico & 1960 & $(\%)$ & 1970 & $(\%)$ & 1980 & $(\%)$ \\
\hline Total & 11332016 & 100.0 & 12955057 & 100.0 & 21941693 & 100.0 \\
& & & & & & \\
Agricultura & 6144930 & 54.2 & 5103519 & 39.0 & 5699971 & 26.0 \\
Minería & 141801 & 1.3 & 180175 & 1.4 & 477017 & 2.1 \\
Construcción & 408402 & 3.6 & 571006 & 4.4 & 1296337 & 6.0 \\
Manufactura & 1556315 & 13.7 & 2169074 & 16.7 & 2575124 & 11.4 \\
Transportación & 398502 & 3.5 & 422098 & 3.2 & 788043 & 4.0 \\
Comercio & 1074593 & 9.5 & 1196878 & 9.2 & 1729296 & 7.8 \\
Servicios & 1525682 & 13.5 & 2564782 & 19.8 & 2823868 & 12.7 \\
Otros & 81791 & 0.7 & 747525 & 5.9 & 6552037 & 29.7
\end{tabular}

FUENTE: III Censo general de población 1960. Dirección general de estadística, SIC, México, 1982.x Censo general de población, 1970. Dirección general de estadística, SIC, México, 1972. Censo general de población, 1980. SPP-INEGI, México, 1984.

NOTA: Porcentajes calculados por el autor. 
CUADRO 5. Estados Unidos, estructura del empleo, 1960, 1970, 1980 (miles).

\begin{tabular}{lrrrrrr}
\hline Sector económico & 1960 & $(\%)$ & 1970 & $(\%)$ & 1980 & $(\%)$ \\
\hline Total & 64639256 & 100.0 & 76553599 & 100.0 & 97639355 & 100.0 \\
& & & & & & \\
Agricultura & 4349371 & 6.7 & 2840488 & 3.7 & 2913598 & 3.0 \\
Minería & 654006 & 1.0 & 630788 & 0.8 & 1028178 & 1.0 \\
Construcción & 3815942 & 6.0 & 4572235 & 6.0 & 5739598 & 6.0 \\
Manufactura & 17513599 & 27.1 & 19837208 & 25.9 & 21914754 & 22.4 \\
Transportación & 4458147 & 6.9 & 5186101 & 6.8 & 7087455 & 7.2 \\
Comercio & 11792636 & 18.2 & 15372880 & 20.1 & 19933926 & 20.4 \\
Servicios & 16361957 & 25.3 & 23912247 & 31.2 & 33874389 & 34.7 \\
Admón. Pública & 3085514 & 4.8 & 4201652 & 5.5 & 5147466 & 5.3 \\
No reportados & 2608085 & 4.0 & & & & \\
& & & & & & \\
\hline
\end{tabular}

FUENTE: U.S. Bureau of the Census. U.S. Census of Population. 1960. U.S. Bureau of the Census. US. Census of Population. 1970. U.S. Bureau of the Census. US. Census of Population. 1980. Vol. 1 Characteristics of the Population, Defailed Population Characteristics for the three periods. NƠTA: Porcentajes calculados por el autor.

económico de la región. El periodo que va de los inicios del siglo a 1960 fue un periodo de acumulación capitalista, crecimiento poblacional, de rápido crecimiento de localidades fronterizas, de expansión urbana y de condiciones marginales para muchos (Fernández, 1977). Como se mencionó arriba, las tasas más altas de crecimiento poblacional ya se habían experimentado. Hasta 1960, la región fronteriza había presentado ritmos de crecimiento ligeramente por encima de los nacionales. $\mathrm{La}$ información existente en cuanto al empleo, hasta 1960, refleja que la estructura regional de la frontera se concentraba en el sector agrícola (45\%). Sin embargo, los sectores secundario y terciario ( $v$. gr. construcción, manufactura, transporte, comercio y servicios) tenian relevancia en la economía regional. La estructura del empleo en los estados fronterizos mexicanos es muy similar a los patrones seguidos en el resto del país. La diferencia notoria es que la region fronteriza muestra mayor equilibrio y crecimiento en todos sus sectores económicos (ver cuadro 6). La estructura del empleo en los estados fronterizos del sur de Estados Unidos, de 1960 a 1980, refleja básicamente un patrón de crecimiento del sector terciario de la economía (cuadro 7). 
CUADRO 6. Estados fronterizos del norte de México*: 1960 , 1970,1980 (miles).

\begin{tabular}{lrrrrrr}
\hline Sector económico & 1960 & $(\%)$ & 1970 & $(\%)$ & 1980 & $(\%)$ \\
\hline Total & 1780565 & 100.0 & 2087455 & 100.0 & 3438431 & 100.0 \\
Agricultura & 801893 & 45.0 & 607570 & 29.0 & 532867 & 15.5 \\
Minería & 38873 & 2.2 & 50868 & 2.5 & 24850 & 0.7 \\
Construcción & 84007 & 4.7 & 121339 & 5.9 & 231145 & 6.7 \\
Manufactura & 264643 & 14.9 & 363230 & 17.4 & 525590 & 15.3 \\
Transportación & 85033 & 4.7 & 86155 & 4.1 & 170837 & 5.0 \\
Comercio & 202606 & 11.5 & 243114 & 11.6 & 383963 & 11.2 \\
Servicios & 276604 & 15.5 & 492098 & 23.6 & 588953 & 17.1 \\
Otros & 26906 & 1.5 & 12108 & 15.8 & 980226 & 28.5 \\
\hline
\end{tabular}

FUENTE: VIII Censo general de población. 1960. Dirección general de estadística, SIC. México, 1982. XI Censo general de población, 1970. Dirección general de estadística, SIC., México, 1972. x Censo general de población, 1980. SPP-INEGI., México, 1984.

NOTA: Porcentajes calculados por el autor

* Estados fronterizos: Baja California, Chihuahua, Nuevó León y Tamaulipas.

CUADRO 7. Estados fronterizos del suroeste de los Estados Unidos*: 1960, 1970, 1980 (miles).

\begin{tabular}{lrrrrrr}
\hline Sector económico & 1960 & $(\%)$ & 1970 & $(\%)$ & 1980 & $(\%)$ \\
\hline Total & 9797706 & 100.0 & 12563111 & 100.0 & 18573758 & 100.0 \\
Agricultura & 615412 & 6.3 & 468442 & 3.7 & 567670 & 3.0 \\
Minería & 160040 & 1.6 & 174383 & 1.4 & 307323 & 1.6 \\
Construcción & 680477 & 7.0 & 792555 & 6.3 & 1280422 & 7.0 \\
Manufactura & 2007907 & 20.5 & 2497596 & 20.0 & 3488144 & 19.0 \\
Transportación & 690896 & 6.9 & 878583 & 7.0 & 1345439 & 7.2 \\
Comercio & 1927768 & 19.7 & 2696215 & 21.4 & 3949686 & 21.2 \\
Servicios & 2716255 & 27.7 & 4276212 & 34.0 & 6693968 & 36.0 \\
Admon. pública & 566996 & 5.8 & 779125 & 6.2 & 941106 & 5.0 \\
Otros & 431955 & 4.5 & & & & \\
\hline
\end{tabular}

FUENTE: U. S. Bureau of the Census, U. S. Census of Population. Vol. 1 Characteristics of the Population, Detailed Population Characteristics. Arizona (parte 4). California (parte 6), Texas (parte 45) y Nuevo México (parte 35). Volúmenes; 1960, 1973 and 1985.

NOTA: Porcentajes calculados por el autor.

- Estados fronterizos: California, Arizona, Nuevo México, y Texas. 
CUADRO 8. Ciudad Juárez, estructura del empleo: 1960, 1970, 1980 (miles).

\begin{tabular}{lrrrrrr}
\hline Sector económico & 1960 & $(\%)$ & 1970 & $(\%)$ & 1980 & $(\%)$ \\
\hline & & & & & & \\
Total & 85989 & 100.0 & 108078 & 100.0 & 204536 & 100.0 \\
Agricultura & 16518 & 19.2 & 9342 & 8.7 & 6366 & 3.1 \\
Minería & 612 & 0.7 & 601 & 0.5 & 276 & 0.1 \\
Construcción & 7730 & 9.0 & 8851 & 8.2 & 14218 & 7.0 \\
Manufactura & 15871 & 18.4 & 19215 & 17.8 & 44586 & 21.8 \\
Transportación & 4689 & 5.5 & 4951 & 4.6 & 11279 & 5.5 \\
Comercio & 15161 & 17.6 & 19149 & 17.8 & 29455 & 14.4 \\
Servicios & 23247 & 27.0 & 37146 & 34.3 & 35359 & 17.3 \\
Otros & 2161 & 2.6 & 8823 & 8.1 & 62997 & 30.8
\end{tabular}

FUENTE: VIIICenso General de Población, 1960. Estado de Chihuahua, vol. 8, DGE, SIC, 1971. IX Censo General de Población, 1970. Estado de Chihuahua, vol. 8, DGE, SIC, 1971. x Censo General de Población, 1980. Estado de Chihuahus, vol. I, tomo 8, SPP-INEGI, 1983.

NOTA: Porcentajes calculados por el autor.

Los cuadros $8,9,10$ y 11 describen las principales diferencias entre Ciudad Juárez-El Paso y Tijuana-San Diego con respecto a la estructura del empleo para el periodo mencionado. Los indicadores nacionales y regionales pueden verse claramente al nivel de las ciudades. Ciudad Juárez tuvo niveles de crecimiento por encima del nacional y también superiores a los que se observaron en el resto de la región fronteriza (ver cuadro 8).

En general, el crecimiento del sector manufacturero (21.8\%) es el indicador más importante en la economía local. Las tasas de crecimiento en la economía de El Paso no estuvieron por encima de las que se observaron en el país o en la región fronteriza del sur de los Estados Unidos, aunque sus tendencias presentan un balance de su economía con el resto de Estados Unicios (ver cuadro 9). La estructura del empleo de Tijuana y Ciudad Juárez observó niveles de crecimiento superiores al nacional e incluso a los del resto de la región fronteriza mexicana; sin embargo, comparada con la de Ciudad Juárez, Tijuana está más concentrada en los secrores secundario y terciario (ver cuadro 10). La economía de San Diego no tuvo un sector manufacturero tan fuerte, pero puede observarse una econonía local más equilibrada, y de igual manera, al comparársele al nivel nacional, manifestó una fuerte tendencia hacia la economía de los servicios (ver cuadro 11). 
CUADRO 9. E Paso, estructura del empleo: 1960, 1970, 1980 (miles).

\begin{tabular}{lrrrrrr}
\hline Sector económico & 1960 & $(\%)$ & 1970 & $(\%)$ & 1980 & $(\%)$ \\
\hline Total & 86946 & 100.0 & 106919 & 100.0 & 167344 & 100.0 \\
& & & & & & \\
Agricultura & 1808 & 2.1 & 1597 & 1.5 & 1899 & 1.2 \\
Minería & 146 & 0.2 & 1401 & 1.3 & 658 & 0.4 \\
Construcción & 6420 & 7.4 & 6976 & 6.5 & 10318 & 6.2 \\
Manufactura & 14127 & 16.2 & 18359 & 17.2 & 31881 & 19.0 \\
Transportación & 9203 & 10.6 & 8363 & 7.8 & 14448 & 8.6 \\
Comercio & 19827 & 22.8 & 24536 & 23.0 & 38298 & 22.9 \\
Servicios & 24115 & 27.7 & 34403 & 32.2 & 57940 & 34.7 \\
Admón. pública & 3054 & 9.6 & 11284 & 10.5 & 11902 & 7.0 \\
Otros & 2946 & 3.4 & & & & \\
& & & & & & \\
\hline
\end{tabular}

FUENTE: Bureau of the Census, US. Census of Population. vol. I, Characteristics of the Population, Detailed Population Characteristics, Texas volume, part 45, 1960, 1973 y 1983.

NOTA: Porcentajes calculados por el autor.

CUADRO 10. Tijuana, estructura del empleo: 1960, 1970, 1980 (miles).

\begin{tabular}{lrrrrrr}
\hline Sector económico & 1960 & $(\%)$ & 1970 & $(\%)$ & 1980 & $(\%)$ \\
\hline Total & 52832 & 100.0 & 89013 & 100.0 & 161087 & 100.0 \\
& & & & & & \\
Agricultura & 10367 & 19.6 & 8176 & 9.2 & 5094 & 3.1 \\
Mineria & 229 & 0.4 & 441 & 0.5 & 124 & 0.1 \\
Construcción & 4106 & 7.7 & 6386 & 7.2 & 10305 & 6.4 \\
Manufactura & 8412 & 16.0 & 18936 & 21.3 & 27075 & 16.8 \\
Transportación & 2785 & 5.3 & 339 & 33.7 & 6685 & 4.2 \\
Comercio & 9355 & 17.7 & 15069 & 17.0 & 26509 & 16.4 \\
Servicios & 13257 & 25.1 & 28695 & 32.2 & 30469 & 19.0 \\
Otros & 4321 & 8.2 & 7917 & 8.9 & 54825 & 34.0 \\
& & & & & & \\
\end{tabular}

FUENTE: VIII Censo General de Población, 1960. Estado de Baja California, vol. 2, DGE, SIC, 1963. IX Censo General de Población, 1970. Estado de Baja California, vol. 2, DGE, sIC., 1971. x Censo General de Población, 1980. Estado de Baja California, vol. I, tomo 2, sPP-INEGI 1983. NOTA: Porcentajes calculados por el autor. 
CUADRO 11. San Diego, estructura del empleo, 1960, 1970, 1980 (miles).

\begin{tabular}{lrrrrrr}
\hline Sector económico & 1960 & $(\%)$ & 1970 & $(\%)$ & 1980 & $(\%)$ \\
\hline Total & 311911 & 100.0 & 430495 & 100.0 & 756400 & 100.0 \\
Agricultura & 9391 & 3.0 & 10209 & 2.4 & 21089 & 2.8 \\
Minería & 543 & 0.2 & 533 & 0.1 & 957 & 0.2 \\
Construcción & 25627 & 8.3 & 28279 & 6.6 & 48732 & 6.4 \\
Manufactura & 72216 & 23.2 & 75464 & 17.5 & 123385 & 16.3 \\
Transportación & 15875 & 5.1 & 23571 & 5.5 & 44352 & 5.8 \\
Comercio & 58862 & 18.8 & 94387 & 22.0 & 163976 & 21.7 \\
Servicios & 89281 & 28.6 & 16042 & 137.2 & 305046 & 40.3 \\
Admón. pública & 28159 & 9.0 & 37631 & 8.7 & 48863 & 6.5 \\
Otros & 11957 & 3.8 & & & & \\
& & & & & & \\
\hline
\end{tabular}

FUENTE: U.S. Bureau of the Census, U.S. Census of Population, vol. I Characteristics of the Population, Deteiled Population Characteristics, volumen de California, parte 6, 1960, 1973 y 1983.

NOTA: Porcentajes calculados por el autor.

\section{EL MARCO REGIONAL DEL ESTUDIO DE LA FRONTERA NORTE}

En cuanto a los orígenes del desarrollo económico de la región fronteriza de México, existen dos explicaciones generalizadas: una que enfatiza el crecimiento poblacional y otra la expansión económica de la región fronteriza. La primera se origina por el enorme flujo de fuerza de trabajo mexicana hacia los Estados Unidos, pues desde principios de siglo la frontera atrajo inmigrantes a la región, lo que provocó, por tanto, un rápido crecimiento poblacional. ${ }^{4} \mathrm{La}$ segunda se deriva del establecimiento del Programa de Industrialización de la Frontera (PIF), que inició en la región fronteriza un periodo de rápida transformación económica nunca antes experimentado. ${ }^{5}$ El primer argumento está fuertemente sustentado y es especialmente evidente al final del Programa Bracero (diciembre de 1964), cuando miles de desempleados se asentaron en las ciudades

4 Autores como Alba (1977, 1982), Fernández (1977), Victoria (1982), Flores Caballero (1982), Bustamante Lemus (1981), Bustamante (1979), Stoddard (1983), Messmacher (1983), comparten esta opinión.

5 La segunda opinión se argumenta por parte de autores como: Baerrensen $(1971,1975)$, Seligson y Williams (1981), Baird y McCaughan (1979), Ayer y Layton (1974), Hansen (1981), Van der Spek (1975), Hendon (1973), Ericson (1970), Femández (1973). 
fronterizas. ${ }^{6}$ La terminación del Programa Bracero no mitigó el constante flujo de inmigrantes a la frontera norte con la expectativa de cruzar la frontera y trabajar en los Estados Unidos. Por el contrario, aun terminado éste, se convirtió en un catalizador para el continuo flujo de inmigrantes mexicanos indocumentados. En este escenario, la frontera se convirtió en un punto de atracción para miles de trabajadores desempleados y así reforzó la creencia de que en gran parte el crecimiento poblacional se originó con este fenómeno.

El segundo argumento responde, probablemente, al supuesto de que las ciudades fronterizas tendrían dificultades para incorporar a esos miles de desempleados a la economía regional. El gobierno mexicano, siguiendo un modelo de industrialización puesto en marcha en los países asiáticos, permitio y promovio el establecimiento de fábricas especializadas en el procesamiento y ensamblado de productos para el mercado estadounidense. En función de estos argumentos, México establecio el PIF, aunque previo a éste y actuando como un precursor del mismo, existieron tres distintos tipos de regulaciones aduanales a lo largo de la frontera del norte del país: la zona de libre comercio, el puerto libre y la zona de exportación ${ }^{7}$ (Baerresen, 1971). Como resultado del PIF, las ciudades ubicadas en la zona fronteriza se convirtieron en zonas procesadoras para la exportación. La legislación requirió la relocalización de las empresas que se debían de dedicar a procesos intermedios en la producción de bienes dentro del PIF y de la zona procesadora para la exportación.

6 El Programa Bracero fue creado por los Estados Unidos y el gobierno mexicano el 23 de julio de 1942, debido a la escasez de mano de obra que surgio por el envolvimiento de los Estados Unidos en la segunds guerra mundial. La producción agrícola se convirtió en estrategia básica para ganar la guerra. El Programa Bracero se mantuvo por espacio de veintidós affos después de la guerra (Baerresen, 1971). Era un programa de trabajo basado en un acuerdo bilateral con México para el empleo temporal de trabajadores en los Estados Unidos, predominantemente en el sector agrícola.

7 Los tres tipos de áreas aduanales a lo largo de la frontera norte de México fueron: la zona de libre comercio, localizada en el oeste de la frontera mexicana; inclúa los estados de Baja California y en aquel entonces el Territorio de Baja California Sur (hoy Baja Californis Sur) y la orilla oeste del estado de Sonora, el cual permitía la entrada de bienes producidos fuera de México exentos de impuestos (Baerresen, 1971). La zona de libre comercio ha existido en la frontera norte de México durante diferentes periodos, gobiemos y localidades a lo largo del norte de Méxica. Fue creada por primera vez en 1860 en el estado de Tamaulipas. Durante ese tiempo la parte oriental de la frontera norte era la de mayor desarrollo (Palacio Nacional, 1893). El puerto libre se conformaba de pequeños enclaves exentos de impuestos que operaban en las zonas de libre comercio, localizadas solamente en Nogales y Agua Prieta en el estado de Sonora. La zona de exportación se localizaba en el lado este de la frontera e incluía las ciudades de Ciudad Juárez, Ciudad Acuña, Piedras Negras, Nuevo Laredo, Reynosa y Matamoros. Todos los bienes que entraran al territorio mexicano estaban sujetos a impuestos de acuerdo a leyes de importación y tarifas; sin embargo, el PIF permitía la importación exenta de impuestos de bienes requeridos y que fueran para procesos de ensamble y para ser enviados fuera del país (Baerresen, 1971). 
Las ventajas proporcionadas por la zona procesadora para la exportación se caracterizaron por exenciones de impuestos y modalidades varias en cuanto a tarifas. Las empresas sólo eran gravadas por el valor agregado, que significaba el bajo costo de la mano de obra que era pagada a una fracción equivalente de lo que se pagaba en los Estados Unidos. Entre las ventajas competitivas ofrecidas por el PIF se encontraban los bajos salarios, gran oferta de mano de obra, proximidad a los mercados del suroeste de Estados Unidos, buenas comunicaciones y facilidades de transportación, facilidades proporcionadas por las zonas procesadoras de exportación, la inexistencia de sindicatos y los parques industriales entre otros beneficios (Baerresen, 1971). Las primeras empresas atraídas por el PIF fueron básicamente industriales, textiles y electrónicas, que ocupaban fundamentalmente la mano de obra femenina, y no empleaban a la fuerza de trabajo masculina que el Programa Bracero había dejado sin empleo.

\section{CONCLUSIONES}

Estas dos concepciones son consideradas como los principales componentes de la formación regional de la frontera norte. La idea esencial a demostrar es que antes del periodo de consolidación regional fronterizo, 1960-1980, se había configurado ya el modelo de desarrollo económico regional en el norte del país, esto es, una economía primaria-terciarizada (véase cuadro 12). Este argumento se refuerza con base en el cuadro 13 del que, tomando los datos y el esquema de regionalización proporcionado por Unikel (1976), se deduce una clasificación funcional de regiones por actividad económica, en la que se ejemplifica la diversidad de la actividad económica de las regiones del norte de México (ver cuadro 13). Por otro lado, el periodo de 1960 a 1980 consolida el desarrollo del sector secundario básicamente en las áreas urbanizadas, y en mucho gracias al rápido proceso poblacional que experimentaron las ciudades fronterizas. De esta manera se explica cómo la migración y el PIF afectaron a las ciudades fronterizas acelerando, por un lado, el proceso de terciarización y el impulso del sector secundario en la actividad económica fronteriza regional. Esto a su vez generó que ciudades fronterizas relativamente pequeñas cambiaran su composición económica, desarrollando en este sentido actividades económicas predominantes de áreas urbanas (ver cuadro 13). ${ }^{8}$

Como ya se ha documentado, la migración y el PIF no establecieron las condiciones necesarias para el desarrollo que ya se había generado. Lo que

8 El rápido proceso de urbanización del norte del país es señalado por Unikel (1976). La clasificación regional es la misma propuesta por Unikel (1976), y se detalla en el cuadro 13. 
CUADRO 12. Estados fronterizos de México*, estructura del empleo: 1940, 1950, 1960 (miles).

\begin{tabular}{lrrrrrr}
\hline Sector económico & 1940 & $(\%)$ & 1950 & $(\%)$ & 1960 & $(\%)$ \\
\hline Total & $763292 * *$ & 100.0 & $1206896 * *$ & 100.0 & $1780565 * * *$ & 100.0 \\
Agricultura & 438647 & 57.4 & 604470 & 50.1 & 801893 & 45.0 \\
Minería & 34702 & 4.5 & 37788 & 3.1 & 38873 & 2.2 \\
Construcción & 20120 & 2.6 & 59259 & 4.9 & 84007 & 4.7 \\
Manufactura & 83185 & 11.0 & 159032 & 13.2 & 264643 & 14.9 \\
Transportación & 34668 & 4.5 & 57939 & 4.8 & 85033 & 4.7 \\
Comercio & 66877 & 8.7 & 95803 & 8.0 & 202606 & 11.5 \\
Servicios & 83886 & 11.0 & 158427 & 13.1 & 276604 & 15.5 \\
\hline
\end{tabular}

FUENTE: VI, VII, VIII Censos Generales de Población, 1940, 1950, 1960. Dirección General de Estadística, SIC, México. NOTA: Porcentajes calculados por el autor.

* Estados fronterizos: Baja California, Sonora, Chihuahua, Nuevo León y Tamaulipas.

** Los totales reflejan la sumatoria más alta debido a que el sector de electricidad, gas, agua, etc. no se consideró como categoría por separado, pero sí se consideró en el total.

*** En este caso se debe considerar que el total refleja además la suma del sector electricidad y "otros", que generalmente los censos los consideran por separados.

es importante destacar con respecto a las dos concepciones aquí tratadas, es que los continuos flujos de inmigrantes a los estados del norte de México que intentan cruzar la frontera, legal o ilegalmente, en busca de empleo en los Estados Unidos, junto con el impacto del PIF, no fueron los factores predominantes en la formación de la región fronteriza. De una economía tradicional basada en las actividades económicas primarias, la frontera norte de México se transformó en una región en donde los sectores secundario y terciario de la economía han empezado a desarrollarse. Como ya se ha descrito, con respecto al desarrollo económico de esta región y su acelerado ritmo de crecimiento poblacional, debe destacarse que cuando los dos fenómenos en cuestión aparecieron, la región fronteriza del norte de México ya había establecido su patrón de desarrollo económico y el crecimiento poblacional ya había alcanzado sus más altos niveles. Sin embargo, estos dos fenómenos aceleraron el proceso de desarrollo, hoy característico de la frontera norte, y su consolidación. El periodo de 1960 a 1980, según se ha argumentado, fue el periodo de estabilización econó- 
mica y de crecimiento demográfico que transformo a la frontera del norte en una de las regiones más industrializadas, urbanizadas y con una economía de servicios más desarrollada entre todas las regiones de México. Lo que es innegable desde esta perspectiva, es que tanto la inmigración como el PIF devinieron en cuestiones de magnitud internacional y la región fronteriza se convirtió en un área conocida en el escenario internacional. Por otro lado, las relaciones con los Estados Unidos y su frontera sur adquirieron importancia considerable y decisiva para la región fronteriza del norte de México.

\section{CUADRO 13. Clasificación funcional de regiones por actividades económicas 1940-1970'.}

\begin{tabular}{ccccc}
\hline & \multicolumn{4}{c}{ Clasificación funcional $^{2}$} \\
Regiones $^{3}$ & 1940 & 1950 & 1960 & 1970 \\
\hline I & D & D & D & D \\
II & D & D & D & D \\
III & D & S & S & B \\
IV & S & B & B & S \\
V & S & U & U & U \\
VI & U & B & U & U \\
VII & D & D & D & D \\
VIII & U & U & U & U \\
\hline
\end{tabular}

FUENTE: Elaboración a partir del cuadro VI-10 de Unikel, et al. (1976:197).

1 La clasificación funcional está elaborada a partir de las actividades económicas predominantes por regiones y entidades. Las actividades económicas que se consideran son agricultura, minería, manufactura, construcción, transporte, comercio, servicios y administración pública. $2 \mathrm{La}$ clasificación funcional se entiende como: U, uniformidades; B, bifuncionales; S, semidiversificadas (hasta 3 ramas de actividad); D, diversificada (cuatro y más ramas de actividad).

3 La clasificación regional propuesta por Unikel (1976:67-8), utilizada aquí, se describe como sigue:

Región I (Noroeste): Baja California Norte, Baja California Sur, Nayarit, Sinaloa y Sonora.

Región II (Norte): Coahuila, Chihuahua, Durango y Nuevo León.

Región III (Golfo): Tamaulipas y Veracruz.

Región IV (Centro-Norte): Aguscalientes, San Luis Potosí y Zacatecas

Región v (Centro-Oeste): Colima, Guanajuato, Jalisco y Michoacán.

Región VI (Centro): Hidalgo, Morelos, Puebla, Querétaro y Tlaxcala.

Región VII (Valle de México): Distrito Federal y Estado de México.

Región VIII (Sur y Sureste): Campeche, Chiapas, Guerrero, Oaxaca, Quintana Roo, Tabasco y Yucatán. 


\section{BIBLIOGRAFIA}

ALBA, Francisco. 1977. "Condiciones y políticas económicas en la frontera norte de México", Natural Resources Journal, vol. 17, no. 4, octubre.

- 1982. "Mexico's Northem Border: A Framework of Reference", Natural Resources Journal, vol. 22, no. 4, octubre.

AYER, Harry W. y Ross Layton. 1974. "The Border Industry Program and the impacts of expenditures by mexican border industry employees on a U. S. border community: An empirical study of Nogales". Annals of Regional Science, vol. 8, (junio).

BAERRESEN, Donald W. 1971. The Border Industrialization Program of Mexico, Lexington, Mass., Lexington Books.

- 1975. "Unemployment and Mexico's Border Industrialization Program". Inter-American Economic Affairs, vol. 29 (otofío).

BAIRD, Peter y Ed McCaughan. 1979. Beyond the Border: Mexico and the U.S. Today. North American Congress on Latin America, (NACLA), New York.

BARKIN, David y Timothy King. 1970. Desarrollo eocnómico regional: enfoque por cuencas hidrológicas de México. México, Siglo XXI Editores.

BASSOLS Batalla, Ángel. 1971. Geografla, subdesarrollo y regionalización. Editorial Nuestro Tiempo, México.

- 1972. El noroeste de México: un estudio geográficoeconómico. Instituto de Investigaciones Económicas, UNAM, México.

- 1979. México: formación de regiones economicas: influencias, factores y sistemas, Instituto de Investigaciones Económicas, Universidad Autónoma de México, México.

- 1982. Realidades y problemas de la geografta en México. Editorial Nuestro Tiempo, México.

BATAILLON, Claude. 1969. Las regiones geogrúficas de México, México, Siglo XXI Editores.

BUSTAMANTE, Jorge A. 1979. "El estudio de la zona fronteriza MéxicoEstados Unidos". Foro Internacional. Vol. XX, no. 3.

BUSTAMANTE Lemus, Carlos. 1981. "Un enfoque urbano regional de la tendencias recientes de los problemas fronterizos México-Estados Unidos". Ponencia presentada en la Primera Conferencia de Impactos Regionales de las Relaciones Económicas México-Estados Unidos, julio 8-11, Guanajuato, México.

CEPAL. 1972. "Estudio regional de México". Comercio Exterior. Vol. XXII, no. 2, marzo. 
COPLAMAR. 1979. Minimos de Bienestar. COPLAMAR, México. 1982. Necesidades esenciales en México: Situacion actual y perspectivas al año 2000. Geografla de la marginación. COPLAMAR y Siglo XXI, México.

CORONA Renterfa, Alfonso. 1983. "Integración industrial de las regiones fronterizas del norte de México a la economía nacional". Estudios fronterizos. Revista del Instituto de Investigaciones Sociales, Universidad Autónoma de Baja California, año I, septiembre-diciembre.

ERICSON, Anna-Stina. 1970. "Mexico's Border Industrialization Program", Monthly Labor Review. Vol. 93, no. 5 (mayo).

FERNANDEZ, Raúl. 1973. "The Border Industrialization Program on the United States-Mexican Border". The Review of Radical Political Economics, vol. v, no. 1.

- 1977. The United States-Mexico Border: A Political Economic Profile. Notre Dame, Il1.: University of Notre Dame Press.

FLORES Caballero, Romeo R. 1982. Evolución de la frontera norte. Facultad de Economía, Centro de Investigaciones Económicas. Universidad Autónoma de Nuevo León, Monterrey.

FRIEDMANN, John, y Rebecca Morales. 1984. "Transborder Planning: A Case of 'Sophisticated Provocation'?", Ponencia presentada en la Conferencia sobre Estudios Fronterizos, Tijuana, B. C. (abril).

GRAIZBORD, Boris y Daniel Hiernaux. 1981. "Algunas consideraciones geográficas para el análisis del espacio fronterizo". Preparado para su presentación en la Primera Conferencia de Impactos Regionales de las Relaciones Económicas México-Estados Unidos. Julio 8-11, Guanajuato, México.

GRAIZBORD, Boris. 1983. "Integración diferencias regionales e interdependencia en la frontera de México con Estados Unidos". Demografla y economia(53) vol. XXVII, no. 1.

GRUPO INTERPARLAMENTARIO MÉXICO-ESTADOS UNIDOS (Mexico-United States Interparliamentary Group). 1962-1982 (De la Segunda Conferencia a la Vigésima Segunda Conferencia). Mexico-United States Interparliamentary Conference. Reportes de las Delegaciones del Congreso y del Senado de los Estados Unidos Americanos, Oficina de Impresiones del Gobiemo, Washington: 1962 a 1982.

HANSEN, Niles. 1981. The Border Economy, Regional Development in the Southwest, Austin, Texas; University of the Texas Press.

- 1983. "European transboundary cooperation and its relevance to the Unites States-Mexico border". Journal of the American Institute of Planners. Vol. 49, no. 3, verano. 
HENDON, Donald W. 1973. "The Border Industrialization Program", Mexican-American review. Vol. 41, no. 7, julio.

JAMES, Dilmus D. 1983. "Trade and Merchandising". En: Ellwyn R. Stoddard, Richard L. Nostrand y Jonathan P. West (eds.), Borderlands Sourcebook: A Guide to the Literature on Northern Mexico and the American Southwest. University of Oklahoma Press: Norman.

LAVELL, A. M. 1972. "Regional Industrialization in Mexico: Some Policy Considerations".Regional Studies. Vol. 6, no. 3, septiembre.

MESSMACHER, Miguel. 1983. La interdependencia en la frontera norte de México. Centro de Investigaciones y Estudios Superiores en Antropología Social, Cuadernos de la Casa Chata, 92.

PALACIO NACIONAL. 1983. Estudios sobre la zona libre en la frontera norte de la república. México, Tipografía de la Oficina Impresora de Estampillas.

ROSS, Stanley R. 1983. Ecology and Development of the Border Region/Ecologia y Desarrollo de la Región Fronteriza. Segunda Reunión de Universidades de México y Estados Unidos sobre Estudios Fronterizos. ANUIES/PROFMEX, México.

SECURITY PACIFIC NATIONAL BANK. 1981. U.S.-Mexican Border Region Economic Report.

SELIGSON, Mitchell A. y Edward J. Williams. 1981. Maquiladoras and Migration Workers in the Mexico-United States Border Industrialization Program. Mexico-US Border Research Program, University of Texas at Austin.

STERN, Claudio. 1973. Las regiones de México y sus niveles de desarrollo socioeconomico, Jornadas 72, El Colegio de México.

- 1967. "Un análisis regional de México", Demografla y economía. Vol. 1, no. 1.

STODDARD, Ellwyn R. (Ed.). 1975-76. "The Status of U.S.-Mexico Borderlands Studies: A Symposium", Social Science Journal. Vol. 12-13, no. 3-1, octubre-enero.

STODDARD, Ellwyn R., Richard L. Nostrand y Jonathan P. West (edi.). 1983. Borderlansd Sourcebook: A Guide to the Literature on Northen Mexico and the American Southwest. University of Oklahoma Press: Norman.

TAMAYO, Jesús. 1980. "Dos problemas del desarrollo económico fronterizo", en: David Barkin, et al. Las Relaciones México-Estados Unidos. Tomo 1, México, Universidad Nacional Autónoma de México y Nueva Imagen.

- 1981. "La integración de la población fronteriza a la producción y al consumo de bienes estadounidenses: ¿Mutua interdependencia 0 
articulación dependiente?". Ponencia, Primer Encuentro sobre Impactos Regionales de las Relaciones México-Estados Unidos, Guanajuato, México, Julio.

TAMAYO, Jesús y José Luis Fernández. 1983. Zonas fronterizas (Mexico-Estados Unidos). Centro de Investigaciones y Docencia Economica. Colección Ensayos 2. México.

TORRES Ramirez, Olga Esther. 1976. "Algunas observaciones sobre la economía de la frontera norte de México". Comercio Exterior, no. 26, diciembre.

UNIKEL, Luis y Edmundo Victoria Mascorro. 1970. "Mediación de algunos aspectos del desarrollo socio-económico de las entidades federativas de México, 1940-1960". Demografla y economía. México, El Colegio de México, Iv, 3(12).

UNIKEL, Luis, Crescencio Ruiz Chiapetto y Gustavo Garza. 1976. El desarrollo urbano de México: diagnóstico e implicaciones futuras. Centro de Estudios Económicos y demográficos. El Colegio de México, México.

VAN DER SPEK, Peter G. 1975. "Mexico's Booming Border Zone: A Magnet for Labor-Intensive American Plants". Inter-American Economic Affairs. Vol. 29, no. 1.

VICTORIA Mascorro, Edmundo. 1982. "Características del desarrollo económico de là franja fronteriza norte de México". Natural Resources Journal. Vol. 22, no. 4, octubre.

XIRAU Icaza, Joaquín y Miguel Díaz. 1976. Nuestra dependencia fronteriza. Archivo del Fondo 48, Fondo de Cultura Económica, México. 